\title{
A Luminous Companion to SGR 1806-20
}

\author{
M. H. van Kerkwijk, S. R. Kulkarni, K. Matthews, G. Neugebauer \\ Palomar Observatory, California Institute of Technology 105-24, Pasadena, CA 91125, USA
}

\begin{abstract}
We have obtained infrared spectra of the star suggested to be the counterpart of the soft $\gamma$-ray repeater (SGR) 1806-20. We found strong emission lines similar to those seen in the spectra of the rare Luminous Blue Variables and $\mathrm{B}[\mathrm{e}]$ stars. A He I absorption line is also seen, from which we infer a spectral type O9-B2. This classification, in combination with the minimum distance of $\gtrsim 6 \mathrm{kpc}$ inferred from its extinction, makes the star one of the most luminous in the Galaxy. We infer that it is a companion to SGR 1806-20, and suggest that the presence of a companion is somehow related to the SGR phenomenon.
\end{abstract}

Subject headings: gamma rays: bursts - stars: individual: SGR 1806-20-stars: neutron - $\mathrm{X}$ rays: stars

${ }^{1}$ This is a preprint of a paper submitted to The Astrophysical Journal (Letters). No bibliographic reference should be made to this preprint. Permission to cite material in this paper must be received from the authors. 


\section{Introduction}

Soft $\gamma$-ray repeaters (SGR) are distinguished from the classical $\gamma$-ray bursters by their recurrence and $\gamma$-ray spectra (Higdon \& Lingenfelter 1990; Norris et al. 1991). Of the three SGRs known, one, SGR 1806-20, is associated with the center-filled, non-thermal radio nebula G 10.0-0.3 (Kulkarni et al. 1994), which has an X-ray source, AX 1805.7-2025, coincident with its peak (Murakami et al. 1994; Cooke 1993). The similarity to what is observed for plerions (pulsar-powered supernova remnants) like the Crab nebula, has led to the hypothesis that SGRs are young pulsars. However, it is unknown why only some pulsars become SGRs, and how a pulsar can produce the brilliant bursts of $\gamma$ rays that are observed. Models involving a companion could address both issues.

In fact, a highly reddened, luminous star (star A) has been suggested to be the stellar counterpart of SGR 1806-20 (Kulkarni et al. 1995, hereafter K95; Vasisht, Frail, \& Kulkarni 1995). In this paper, we present spectroscopic observations of this star.

\section{Observations}

A K-band spectrum of star A was kindly taken for us in August, 1994, by our colleagues L.J. Smith and P.A. Crowther, using the Cooled Grating Spectrograph 4 attached to the United Kingdown Infrared Telescope (UKIRT) on Mauna Kea, Hawaii. The spectrum covers $2.02-2.22 \mu \mathrm{m}$ with a spectral resolution $\lambda / \Delta \lambda$ of $\sim 700$. Atmospheric and instrumental effects were removed by division by a spectrum of HR 7377 (with its H I Bry line artificially removed). Flux calibration was done by scaling the spectrum to produce the K-band magnitude from K95. The reduced spectrum, reproduced in Figure 1, shows a number of emission features arising from both low and high-excitation ions (Table 1).

The emission lines are reflected as wiggles in lowresolution $(\lambda / \Delta \lambda \simeq 75)$ grism spectra taken earlier with the Hale 200-inch telescope on Palomar Mountain. These $\mathrm{H}$ and K-band spectra were used by K95 to argue that star A was not a late-type luminous star. New J and H-band spectra were obtained in October, 1994, with the same instrumental setup that was used by K95, except that a new $256 \times 256 \mathrm{InSb}$ array was used, and that the slit was narrowed to 0.5 to double the spectral resolution. All spectra were corrected for telluric and instrumental features using spectra of HR 7643, and flux-calibrated by scaling to

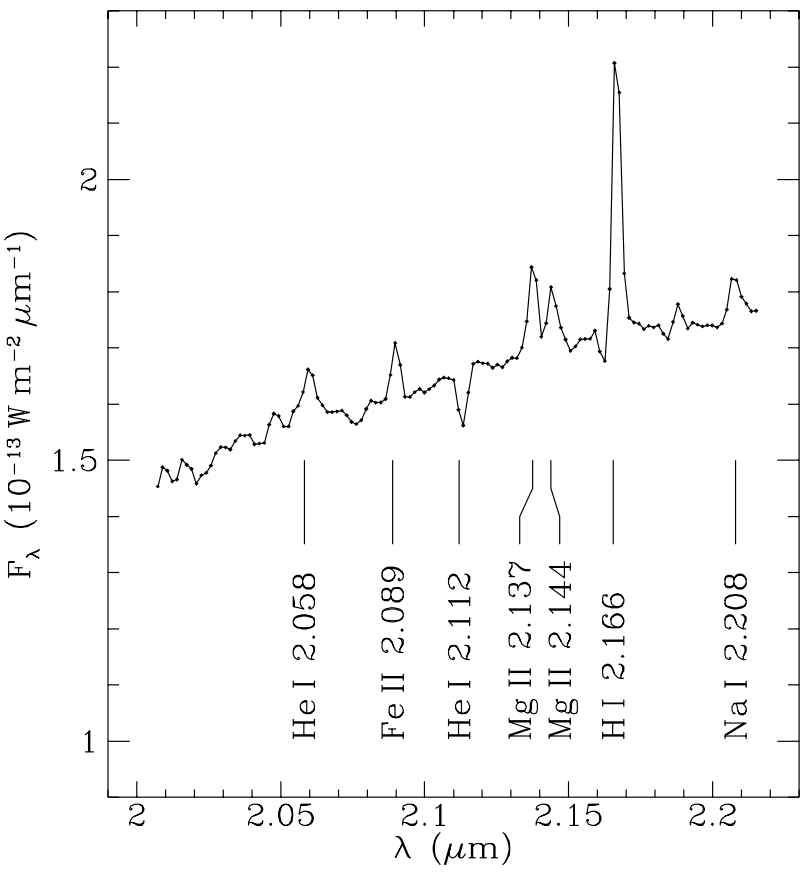

Fig. 1.- The 2.02-2.22 micron UKIRT spectrum obtained on 19 August 1994, and suggested identifications of the emission and absorption features. The wiggles seen around and shortward of He I $\lambda 2.058$ are due to residual telluric $\mathrm{CO}_{2}$ features. A possibly significant feature with a $\mathrm{P}$ Cygni profile is present at $2.188 \mu \mathrm{m}$. If real, it could be due to He II $\lambda 2.189$.

the observed magnitudes from K95. The spectra, reproduced in Figure 2, show a number of additional emission features (Table 1).

\section{Interpretation of the spectra}

The spectra of star A are similar to those observed for luminous blue variables (LBVs), such as $\eta$ Car, and B[e] stars (Allen, Jones, \& Hyland 1985; McGregor et al. 1988a, b; Hamann et al. 1994). Similar emission lines are also observed in some young stellar objects (YSOs), but for the two K-band spectra that we could find, of the Becklin-Neugebauer (B-N) object (Scoville, Kleinmann, \& Hall 1983) and of LkH $\alpha 101$ (Simon \& Cassar 1984), the line strengths and ratios are very different from what we observe. In addition, for the B-N object, lines of $\mathrm{H}_{2}$ are observed, which are not present in our spectra.

One can infer from the presence of strong emission lines that there is a substantial amount of circumstel- 

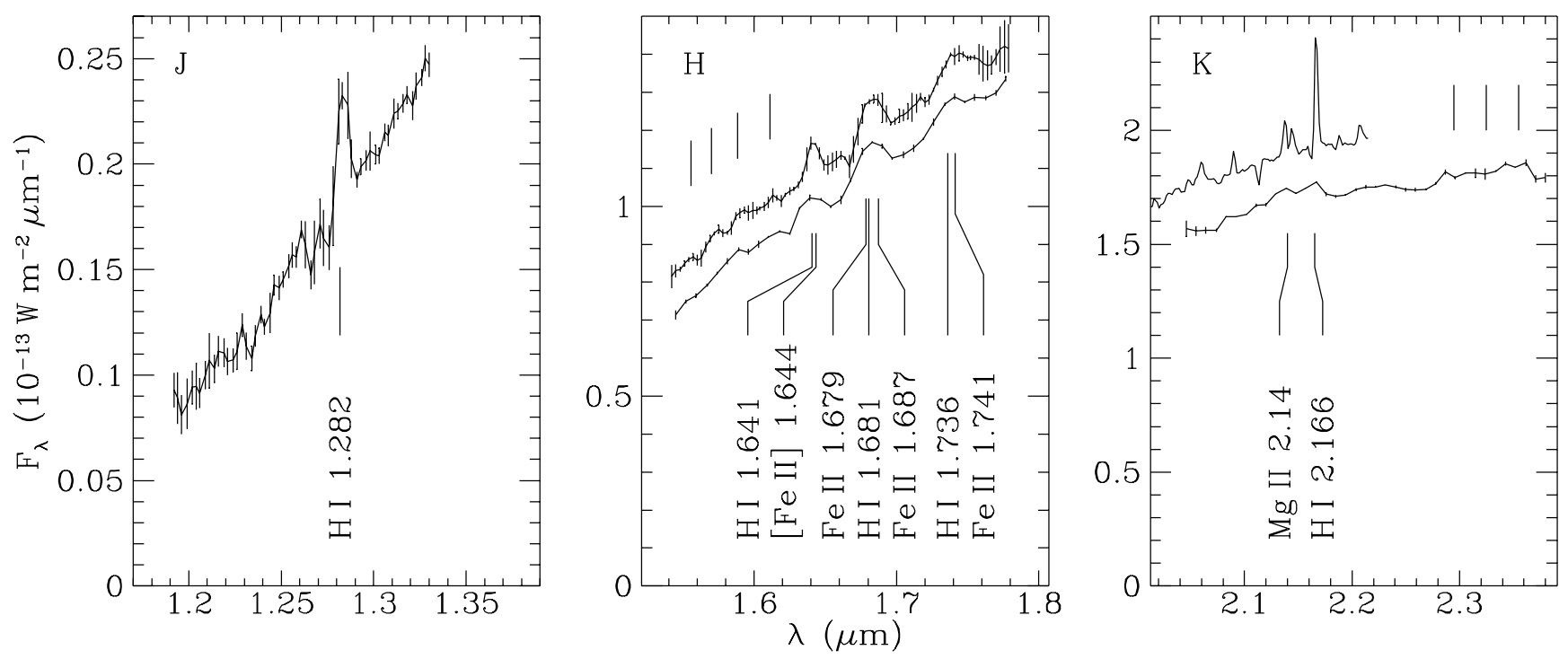

Fig. 2.- The J, H and K-band Palomar spectra. The low-resolution spectra in the $\mathrm{H}$ and $\mathrm{K}$ bands (lower curves) were obtained on 6 October 1993, and the higher-resolution ones in $\mathrm{J}$ and $\mathrm{H}$ (upper curve; offset by 0.1 ) on 18 October 1994. The UKIRT K-band spectrum (upper curve; offset by 0.2), is reproduced for comparison. Suggested identifications of the emission features are given. Above the H-band spectra, also the rest wavelengths of some higher members of the Brackett series are indicated, which seem to be in emission as well. Above the K-band spectrum, the heads of the first-overtone $\mathrm{CO}$ bands are indicated. They may be in emission, but this needs to be confirmed. Possibly significant emission is also seen blueward of $\mathrm{Pa} \beta$. This may be due partly to [Fe II] 1.257.

lar matter in the system. This could be either in the form of a disk or an outflowing wind. In Figure 1, $\operatorname{Br} \gamma$ appears to have a blueshifted absorption component, i.e., a P Cygni profile. If so, it would indicate an outflow velocity of $\sim 500 \mathrm{~km} \mathrm{~s}^{-1}$. The width of the emission lines is consistent with such an outflow velocity. It is similar to the velocity outflow seen in $\eta$ Car (Hamann et al. 1994), but much larger than the outflow velocity seen in the B-N object and $\mathrm{LkH} \alpha 101$ (Scoville et al. 1983; Simon \& Cassar 1984).

Since sodium is not expected to be neutral at the temperatures necessary to excite helium, the simultaneous presence of both a $\mathrm{NaI}$ and a $\mathrm{HeI}$ line in the spectrum may be surprising. It probably indicates that there are two physically distinct circumstellar regions, e.g., a disk and a radiation-driven wind (Allen et al. 1985; McGregor et al. 1988b; Scoville et al. 1983).

The presence of He I $\lambda 2.113$ in absorption indicates that the star proper is hot. From the equivalent width, one can infer a spectral type B2-O9 (Hanson $\&$ Conti 1994). For such a star, the intrinsic IR colors are essentially neutral $\left((J-H)_{0}=(H-K)_{0} \simeq\right.$
0 mag.). However, for some LBVs (McGregor et al. 1988a, b) and for all B[e] stars (Zickgraf 1990), the intrinsic colors are much redder $\left((J-H)_{0} \simeq 0.5\right.$ mag.; $(H-K)_{0} \simeq 1.0$ mag. $)$, indicating the presence of hot dust $(T \simeq 1000 \mathrm{~K})$. We can test for the presence of dust by dereddening the observed colors for star A $((J-H)=3.1$ mag.; $(H-K)=1.8$ mag.; K95). Although the interstellar extinction law is rather uncertain, with values for $E_{J-H} / E_{H-K}$ ranging from 1.6 to 2.1 (Mathis 1990), we find that the presence of dust is excluded. Thus, the object is most likely not a $\mathrm{B}[\mathrm{e}]$ star.

Assuming neutral colors and using an extinction curve appropriate for the Galactic Centre (from Rieke \& Lobofsky 1985; for this curve, $E_{J-H} / E_{H-K}=1.7$ ), we infer a foreground extinction $A_{V}$ of $\sim 28$ mag. An almost identical value is deduced from model fitting of the X-ray data of AX 1805.7-2025 (Sonobe et al. 1994). K95 suggested that this extinction arises in a molecular cloud in the direction of SGR 1806-20, located at a distance of about $6 \mathrm{kpc}$. If so, then given the minimum distance of $6 \mathrm{kpc}$, the absolute J magnitude of star A would be $<-8.5$ mag. Since the spec- 
TABLE 1

Line Identifications and Equivalent Widths

\begin{tabular}{cclcc}
\hline \hline $\begin{array}{c}\lambda_{\text {UKIRT }} \\
(\mu \mathrm{m})\end{array}$ & $\begin{array}{c}\lambda_{200^{\prime \prime}} \\
(\mu \mathrm{m})\end{array}$ & \multicolumn{1}{c}{ Identification } & $\begin{array}{c}\lambda_{\text {lab }} \\
(\mu \mathrm{m})\end{array}$ & $\begin{array}{c}\text { EW } \\
(\AA)\end{array}$ \\
\hline$\ldots$ & $1.2830(15)$ & H I $5-3(\mathrm{~Pa} \beta)$ & 1.2818 & $-22(4)$ \\
$\ldots$ & $1.6406(15)$ & H I $12-4(\mathrm{Br} 12)$ & 1.6407 & $-9.4(20)$ \\
& & Fe II] $a^{4} F_{9 / 2}-a^{4} D_{7 / 2}$ & 1.6436 & \\
& & Fe II $z^{4} F_{9 / 2}-c^{4} F_{7 / 2}$ & 1.6787 & \\
$\ldots$ & \multirow{2}{*}{$1.6810(20)$} & H I $11-4(\mathrm{Br} 11)$ & 1.6806 & $-15.0(20)$ \\
& & Fe II $z^{4} F_{9 / 2}-c^{4} F_{9 / 2}$ & 1.6873 & \\
$\ldots$ & $1.7401(20)$ & H I $10-4(\mathrm{Br} 10)$ & 1.7362 & $-14(3)$ \\
$2.0591(10)$ & $\ldots$ & He II $z^{4} F_{7 / 2}-c^{4} F_{7 / 2}$ & 1.7414 & \\
$2.0893(5)$ & $\ldots$ & Fe II $z^{4} F_{3 / 2}-c^{4} F_{5 / 2}$ & 2.0888 & $-2.1(4)$ \\
$2.1125(5)$ & $\ldots$ & He I $2 p^{3} P^{0}-4 s^{3} S$ & 2.1126 & $+1.8(4)$ \\
$2.1370(5)$ & & Mg II $5 p^{2} P_{3 / 2}-5 s^{2} S_{1 / 2}$ & 2.1375 & $-3.8(4)$ \\
$2.1437(5)$ & $2.137(4)$ & Mg II $5 p^{2} P_{1 / 2}-5 s^{2} S_{1 / 2}$ & 2.1438 & $-2.7(4)$ \\
$2.1660(5)^{\mathrm{a}}$ & $2.165(4)$ & H I $7-4(\mathrm{Br} \gamma)$ & 2.1655 & $-10.8(6)^{\mathrm{a}}$ \\
& $\ldots$ & Na I $4 p^{2} P_{3 / 2}^{0}-4 s^{2} S_{1 / 2}$ & 2.2056 & $-2.4(3)$ \\
$2.2071(5)$ & $\ldots$ & Na I $4 p^{2} P_{1 / 2}^{0}-4 s^{2} S_{1 / 2}$ & 2.2083 & \\
\hline & & & & \\
\hline
\end{tabular}

${ }^{\text {a }}$ The listed values are for the emission component. The possible absorption component is at 2.1620(15) $\mu \mathrm{m}$ and has an equivalent width of $0.7(3) \AA$

NotE.-All wavelengths are in vacuo. EW is equivalent width. Numbers in parentheses indicate $90 \%$ confidence levels in the final decimal place.

tral type is $\mathrm{B} 2$ or earlier, the bolometric magnitude would be $<-10.4 \mathrm{mag}$. and the bolometric luminosity $>10^{6} L_{\odot}$. Only the most massive stars, including LBVs such as $\eta$ Car, have such high luminosities (Humphreys \& Davidson 1994).

\section{Discussion and Conclusion}

From the emission-line spectrum and the inferred luminosity it follows that star A is most likely a star similar to a LBV. It can only be classified as a 'candidate LBV', however, since there is as yet no evidence for the large variability that is one of the defining characteristics of LBVs (Humphreys \& Davidson 1994).

Extrapolating from the LMC (Humphreys \& Davidson 1979), we estimate that there are only a couple of hundred stars of similar luminosity in our Galaxy.
The probability of a chance coincidence with the radio peak (1" ; Vasisht et al. 1995) or even the quiescent X-ray source (11"; Cooke 1993) is exceedingly small. We conclude that star A is a very luminous stellar counterpart of SGR 1806-20.

The impact of this new finding on our understanding of the nature of SGRs is unclear. If the SGR outbursts originate on or near young, rapidly rotating neutron stars, as seems likely given the short timescales and high luminosities (Higdon \& Lingenfelter 1990: Norris et al. 1991), the neutron star cannot be an isolated object. If interaction with the companion causes the $\gamma$-ray outbursts, this could explain why only some young neutron stars show bursts, while others, like the Crab, do not.

However, in the error box of the X-ray counterpart of SGR 0526-66, associated with the supernova rem- 
nant (SNR) N49 in the LMC, there is no star similar to a LBV or B[e] star (Rothschild, Kulkarni \& Lingenfelter 1994: Fishman, Duthie, \& Dufour 1981). From a CCD image, taken with the 2.3-m telescope at Siding Spring Observatory in a band centered on $6620 \AA$ (relatively free of SNR emission lines), we infer a limit on the absolute magnitude $M_{6620}>-0.7 \mathrm{mag}$. Hence, any binary model for SGRs must not rely on the presence of a massive stellar companion.

For SGR 1806-20, the quiescent radio (Vasisht et al. 1995) and X-ray (Sonobe et al. 1994) emission do not show the large variations typically observed in accreting systems. This suggests that little interaction takes place in quiescence, perhaps because the pulsar wind and magnetic field prevent matter from accreting. It could be that the quiescent emission is due to the pulsar spinning down, and that the companion merely acts as a trigger or a catalyst for the $\gamma$-ray bursts. Models attributing SGR flashes to accretion of planetesimals or asteroids (see Higdon \& Lingenfelter 1990; Katz, Toole, \& Unruh 1994 and references therein) may not be as far fetched as had been thought before.

We thank L. J. Smith and P. A. Crowther for obtaining the UKIRT spectrum for us, T. R. Geballe for useful discussions about the line identifications, and J. F. Bell and M. A. Dopita for obtaining the CCD image of N49. UKIRT is operated by The Observatories, on behalf of the UK Particle Physics and Astronomy Council. M.H.v.K. is supported by a NASA Hubble Fellowship, S.R.K. by grants from the US NSF, NASA and the Packard Foundation, and infrared astrophysics at Caltech by a grant from the NSF.

\section{REFERENCES}

Allen, D. A., Jones, T. J., \& Hyland, A. R. 1985, ApJ, 291, 280

Cooke, B. A. 1993, Nature, 366, 413

Fishman, G. J., Duthie, J. G., \& Dufour, R. J. 1981, Ap\&SS, 75, 135

Hamann, F., DePoy, D. L., Johansson, S., \& Elias, J. 1994, ApJ, 422, 626

Hanson, M. M., \& Conti, P. S. 1994, ApJ, 423, L139

Higdon, J. C. \& Lingenfelter, R. E. 1990, ARA\&A, 28,401

Humphreys, R. M., Davidson, K., 1979, ApJ, 232, 409

Humphreys, R. M., Davidson, K. 1994, PASP, 106, 1025
Katz, J. I., Toole, H. A., \& Unruh, S. H. 1994, ApJ, 437, 727

Kulkarni, S. R., Frail, D. A., Kassim, N. E., Murakami, T., \& Vasisht, G. 1994, Nature, 368, 129

Kulkarni, S. R., Matthews, K., Neugebauer, G., Reid, I. N., van Kerkwijk, M. H., \& Vasisht, G. 1994, ApJ (in press; K95)

Mathis, J. S. 1990, ARA\&A, 28, 37

McGregor, P. J., Hyland, A. R., \& Hillier, D. J. 1988a, ApJ, 324, 1071

McGregor, P. J., Hillier, D. J., \& Hyland, A. R. 1988b, ApJ, 334, 639

Murakami, T., Tanaka, Y., Kulkarni, S. R., Ogasaka, Y., Sonobe, T., Ogawara, Y., Aoki, T. \& Yoshida, A. 1994, Nature, 368, 127

Norris, J. P., Hertz, P., Wood, K. S., \& Kouveliotou, C. 1991, ApJ, 366, 240

Rieke, G., \& Lobofsky, M.J. 1985, ApJ, 288, 618

Rothschild, R. E., Kulkarni, S. R., \& Lingenfelter, R. E. 1994, Nature, 368, 432

Scoville, N., Kleinmann, S. G., Hall, D. N. B., \& Ridgway, S. T. 1983, ApJ, 275, 201

Simon, M., \& Cassar, L. 1984, ApJ, 283, 179

Sonobe, T., Murakami, T., Kulkarni, S. R., Aoki, T., \& Yoshida, A. 1994, ApJ, 436, L23

Vasisht, G., Frail, D. A., \& Kulkarni, S. R. 1995, ApJ (in press)

Zickgraf, F.-J. 1990, in Angular Momentum and Mass Loss for Hot Stars (ed. Davidson, K., Moffat, A. F. J., \& Lamers, H. J. G. L. M.) 245

This 2-column preprint was prepared with the AAS LATEX macros v3.0. 